\title{
Average Consensus in Networks of Neutral Dynamical Agents with Fixed and Switching Topologies
}

\author{
Yingying Wu and Yuangong Sun \\ School of Mathematical Sciences, University of Jinan, Jinan, Shandong 250022, China \\ Correspondence should be addressed to Yuangong Sun; sunyuangong@163.com
}

Received 15 March 2015; Revised 5 August 2015; Accepted 12 August 2015

Academic Editor: Akio Matsumoto

Copyright (C) 2015 Y. Wu and Y. Sun. This is an open access article distributed under the Creative Commons Attribution License, which permits unrestricted use, distribution, and reproduction in any medium, provided the original work is properly cited.

\begin{abstract}
This paper addresses the average consensus problem of neutral multiagent systems in undirected networks with fixed and switching topologies. For the case of fixed topology, necessary and sufficient conditions to average consensus are established by decoupling the neutral multiagent system in terms of the eigenvalues of the Laplacian matrix. For the case of switching topology, sufficient conditions to average consensus are given in terms of linear matrix inequalities to determine the allowable upper bound of the time-varying communication delay. Finally, two examples are worked out to explain the effectiveness of the theoretical results.
\end{abstract}

\section{Introduction}

In the last few years, more and more researchers have focused their attention on the study of multiagent systems due to many applications in unmanned aerial vehicles, satellite cluster, automated highways, and mobile robots. In all cases, the aim is to control a group of agents connected through a wireless network. Many profound theoretical results have been established for cooperative control of multiagent systems. In cooperative control of multiagent systems, an important issue is to design appropriate control strategies or protocols based on local information such that the group of dynamic agents can reach an agreement on certain quantities of interest. Such a problem is usually called a consensus problem, which has attracted many people in recent years.

Consensus problem has a long history in the field of computer science. In the field of control theory, a discretetime model of $n$ agents all moving in the plane with the same speed was proposed by Vicsek et al. [1]. A theoretical explanation of the consensus behavior in the Vecsek model was put forward by Jadbabaie et al. [2], where the alignment of a network of agent with switching topologies that are periodically connected was analyzed. Olfati-Saber and Murray [3] discussed the consensus problem for networks of dynamic agents with switching topologies and time delays in a continuous-time model by defining a disagreement function. Some more relaxable consensus conditions were presented by Ren and Beard [4] under dynamically changing interaction topologies.

In recent years, more and more consensus results with single integrator models were established. Xiao and Wang studied the consensus problem of discrete-time multiagent systems with time delay $[5,6]$. Sun et al. $[7,8]$ studied the average consensus problem in undirected networks of multiagent systems with fixed and switching topologies as well as multiple time-varying communication delays. Yu et al. [9] investigated the average consensus of multiagent systems with the problem of packet losses when both the first-order neighbors' information and the second-order neighbors' information are used. The consensus problem in directed networks via nonlinear protocols was premeditated by Liu et al. [10], and consensus of nonlinear multiagent systems with observer-based protocols was studied by Hu et al. [11].

Unlike the single integrator case, a spanning tree is a necessary rather than a sufficient condition for consensus seeking with double integrator dynamics. However, the extension of consensus algorithms from the first-order case to the second-order case is nontrivial [12]. The general consensus protocol of multiagent systems with second-order dynamics was investigated by Zhu et al. [13], where necessary and sufficient conditions to consensus were given. Tian and Liu [14] considered the robust consensus of second-order multiagent 
systems with different input delays and asymmetric interconnection perturbations based on the frequency-domain analysis method. Yu et al. [15] investigated the second-order consensus for nonlinear multiagent systems with directed topologies. Song et al. [16] studied the second-order leaderfollowing consensus of nonlinear multiagent systems by pinning control. Consensus algorithms designed for double integrator dynamics were provided by Abdessameud and Tayebi [17]. Zheng et al. [18] investigated the consensus problem of heterogeneous multiagent systems. Some sufficient conditions to consensus were established for both cases of undirected graphs and leader-following networks.

It is well known that neutral systems play an important role in engineering area. If the agent's dynamical equation takes the neutral form, what about the consensus of multiple agents in networks? To the best of our knowledge, consensus problem of neutral multiagent systems is not resolved by now. Therefore, it is necessary to study the consensus of neutral multiagent systems, which is the main purpose of this paper. We make the following contributions. Firstly, average consensus for neutral multiagent systems in undirected networks is proposed. Secondly, the convergence of the given proposed protocols is analyzed, and sufficient and necessary or sufficient conditions to average consensus of neutral multiagent systems have been established.

The remainder of this paper is organized as follows. In Section 2, graph theory and some important definitions and lemmas are given. The neutral multiagent system model is also established. The average consensus analysis of the neutral multiagent system with fixed and switching topologies is given in Section 3. Two examples are provided in Section 4. Finally, conclusions are drawn in Section 5.

\section{Preliminaries and Problem Statement}

As usual, the communication network is modeled through an undirected weighted graph $G=(\mathscr{V}, \mathscr{E}, \mathscr{A})$ defined by a set of nodes $\mathscr{V}=\left\{v_{1}, v_{2}, \ldots, v_{n}\right\}$, a set of edges $\mathscr{E} \subset \mathcal{N} \times \mathcal{N}$, and a weighted adjacency matrix $\mathscr{A}=\left[a_{i j}\right]$ with nonnegative adjacency elements $a_{i j}$. The node indexes belong to a finite index set $\mathcal{N}=\{1,2, \ldots, n\}$, and $v_{i}$ represents the $i$ th agent. An edge of $G$ is denoted by $e_{i j}=\left(v_{i}, v_{j}\right)$. The adjacency elements associated with the edges of the graph are positive, that is, $e_{i j} \in$ $\mathscr{E}$ if and only if $a_{i j}>0$. Moreover, we assume $a_{i i}=0$ and $a_{i j}=$ $a_{j i}$, for $i, j \in \mathcal{N}$. The set of neighbors of node $v_{i}$ is denoted by $N_{i}=\left\{v_{j} \in \mathscr{V}:\left(v_{i}, v_{j}\right) \in \mathscr{E}\right\}$. An undirected graph is called connected if any two distinct nodes of the graph can be connected via a path that follows the edges of the graph. The Laplacian matrix $L(G)=\left[l_{i j}\right]$ of the graph is defined by

$$
l_{i j}= \begin{cases}-\sum_{j=1, j \neq i}^{n} a_{i j}, & j=i, \\ a_{i j}, & j \neq i .\end{cases}
$$

It is easy to see that $L(G)$ is symmetric and $\mathbf{1}^{T} L(G)=$ $L(G) \mathbf{1}=\mathbf{0}$, where $\mathbf{1}$ and $\mathbf{0}$ are appropriate dimensional vectors with entries 1 and 0 . We now define an operator $\Delta$ as

$$
\Delta x=L x, \quad x \in \mathbb{R}^{n} \text {. }
$$

Let $H_{1}$ represent the space collecting all vectors in $\mathbb{R}^{n}$ with zero average. The eigenstructure of the operator $\Delta$ is completely characterized by the next lemma proved in Bensoussan and Menaldi [19].

Lemma 1. (a) The operator $\Delta: H_{1} \rightarrow H_{1}$ is symmetric. It has $n-1$ strictly negative eigenvalues and the corresponding eigenvectors form an orthogonal basis for $H_{1}$; (b) for $x \in \mathbb{R}^{n}$, $\Delta x=0$ if and only if $x \in H_{1}^{\perp}$.

Lemma 1 shows that the operator $\Delta$ or the Laplacian matrix $L(G)$ is invertible on the subspace $H_{1}$. Now, consider the following neutral multiagent system model:

$$
\dot{x}_{i}(t)+c \dot{x}_{i}(t-\sigma)=u_{i}(t), \quad i \in \mathcal{N},
$$

where $x_{i}(t) \in \mathbb{R}$ and $u_{i}(t) \in \mathbb{R}, i \in \mathcal{N}, t \in \mathbb{R}_{+}$, denote the state and the control input of the $i$ th agent at time $t$, respectively, and $\sigma>0$ and $c$ are constants. Consider the delayed Laplacian protocol as follows:

$$
u_{i}(t)=\sum_{v_{j} \in N_{i}} a_{i j}\left[x_{j}(t-\tau(t))-x_{i}(t-\tau(t))\right], \quad i \in \mathcal{N},
$$

where the time-varying delay $\tau: \mathbb{R}_{+} \rightarrow \mathbb{R}_{+}$is a piecewise continuous function satisfying $0 \leq \tau(t) \leq h$, for $h>0$. Then, the neutral multiagent system takes the form

$$
\begin{aligned}
\dot{x}_{i}(t) & +c \dot{x}_{i}(t-\sigma) \\
& =\sum_{v_{j} \in N_{i}} a_{i j}\left[x_{j}(t-\tau(t))-x_{i}(t-\tau(t))\right], \quad i \in \mathcal{N} .
\end{aligned}
$$

Rewrite (5) in the following operator form:

$$
\dot{X}(t)+c \dot{X}(t-\sigma)=\Delta X(t-\tau(t)),
$$

where $X(t)=\left[x_{1}(t), x_{2}(t), \ldots, x_{n}(t)\right]^{T}$.

As a result of the supposed piecewise continuity of the delay, the Cauchy problem associated with (6) is defined because it admits unique solution, depending on the initial condition. Set $\mu=\max \{\sigma, h\}$. The initial condition may be given by a continuously differentiable function $X(t)=\widetilde{X}(t)$ defined on $[-\mu, 0]$. In this paper, assume without loss of generality that $x_{i}(t) \equiv x_{i}(0)$, for $t \in[-\mu, 0]$.

The average consensus for the neutral multiagent system (6) is defined as follows.

Definition 2. The neutral multiagent system (6) is said to achieve consensus if

$$
\lim _{t \rightarrow \infty} X(t)=\alpha \mathbf{1}
$$

where $\alpha$ is a constant. If $\alpha=(1 / n) \sum_{i=1}^{n} x_{i}(0)$, the neutral multiagent system (6) is said to achieve average consensus.

Remark 3. For the neutral multiagent system (6), once it achieves consensus, it must achieve average consensus since the involved graph is undirected. In fact, if (7) holds, noting that $\mathbf{1}^{T} \Delta=0$, we have

$$
\mathbf{1}^{T}[\dot{X}(t)+c \dot{X}(t-\sigma)]=\mathbf{1}^{T} \Delta X(t-\tau(t))=0,
$$


which implies that $\mathbf{1}^{T}[X(t)+c X(t-\sigma)]$ is an invariant quantity. Therefore,

$$
\mathbf{1}^{T}[X(t)+c X(t-\sigma)]=\mathbf{1}^{T}[X(0)+c X(-\sigma)],
$$

$$
t \geq 0 \text {. }
$$

Since $X(t) \equiv X(0)$, for $t \in[-\sigma, 0]$, we have that $\alpha=$ $(1 / n) \sum_{i=1}^{n} x_{i}(0)$.

By Lemma 1 and the following decomposition $X=X_{1}+$ $\bar{X}$, where $X_{1} \in H_{1}, \bar{X} \in H_{1}^{\perp}$, we can easily get the following lemma.

Lemma 4. The function $X$ is a solution of (6) if and only if $X_{1}$ and $\bar{X}$ are solutions of the following:

$$
\begin{aligned}
\dot{X}_{1}(t)+c \dot{X}_{1}(t-\sigma) & =\Delta X_{1}(t-\tau(t)), \\
\dot{\bar{X}}(t)+c \dot{\bar{X}}(t-\sigma) & =0 .
\end{aligned}
$$

Remark 5. By Lemma 4, we know that the spaces $H_{1}$ and $H_{1}^{\perp}$ are positively invariant for (6). Furthermore, by Remark 3, the average state $\bar{X}$ is a constant in time and equals $(1 / n) \sum_{i=1}^{n} x_{i}(0)$. Thus, the problem of average consensus for (6) is simplified to prove that $X_{1}(t) \rightarrow 0$ as $t \rightarrow \infty$; that is, the zero solution of (10) is asymptotically stable.

\section{Main Results}

In this section, we will consider the average consensus of neutral multiagent systems with fixed and switching topologies, respectively.

\subsection{Networks with Fixed Topology}

Theorem 6. Let $G=(\mathscr{V}, \mathscr{E})$ be an undirected connected weighted graph. The neutral multiagent system (6) achieves average consensus if and only if all the following scalar differential equations

$$
\begin{aligned}
\dot{\alpha}_{k}(t)+c \dot{\alpha}_{k}(t-\sigma)=\lambda_{k} \alpha_{k}(t-\tau(t)) & \\
k & \\
k & =1,2, \ldots, n-1
\end{aligned}
$$

are asymptotically stable, where $\lambda_{1} \leq \lambda_{2} \leq \cdots \leq \lambda_{n-1}<0$ are eigenvalues of the operator $\Delta$.

Proof. Let $\left\{\varphi_{k}\right\}_{k=1}^{n-1}$ be a set of eigenfunctions of $\Delta$ corresponding to the eigenvalues $\left\{\lambda_{k}\right\}_{k=1}^{n-1}$ that forms a basis for $H_{1}$. Then, there exist suitable functions $\alpha_{k}: \mathbb{R} \rightarrow \mathbb{R}$ such that

$$
X_{1}(t)=\sum_{k=1}^{N-1} \alpha_{k}(t) \varphi_{k}
$$

By multiplying $\varphi_{m}(m=1,2, \ldots, n-1)$ on both sides of the first equation in (10), we have that the left-hand side and the right-hand side of (10), respectively, take the following forms:

$$
\begin{aligned}
& \left\langle\varphi_{m}, \dot{X}_{1}(t)+c \dot{X}_{1}(t-\sigma)\right\rangle \\
& =\left\langle\varphi_{m}, \sum_{k=1}^{n-1}\left(\dot{\alpha}_{k}(t)+c \dot{\alpha}_{k}(t-\sigma)\right) \varphi_{k}\right\rangle \\
& =\dot{\alpha}_{m}(t)+c \dot{\alpha}_{m}(t-\sigma), \\
& \left\langle\varphi_{m}, \Delta X_{1}(t-\tau(t))\right\rangle=\left\langle\varphi_{m}, \sum_{k=1}^{n-1} \alpha_{k}(t-\tau(t)) \Delta \varphi_{k}\right\rangle \\
& =\left\langle\varphi_{m}, \sum_{k=1}^{n-1} \alpha_{k}(t-\tau(t)) \lambda_{k} \varphi_{k}\right\rangle \\
& =\lambda_{m} \alpha_{m}(t-\tau(t)) .
\end{aligned}
$$

By (14) and (15), one can obtain

$$
\begin{array}{r}
\dot{\alpha}_{m}(t)+c \dot{\alpha}_{m}(t-\sigma)=\lambda_{m} \alpha_{m}(t-\tau(t)), \\
\quad m=1,2, \ldots, n-1 .
\end{array}
$$

Therefore, the neutral multiagent system (6) can achieve average consensus; that is, (10) is asymptotically stable, if and only if all equations (16) are asymptotically stable for $m=$ $1, \ldots, n-1$. The proof of Theorem 6 is completed.

For the particular case $\tau(t) \equiv \sigma$, the following lemma is required.

Lemma 7 (see [20]). The following scalar neutral differential equation

$$
\dot{x}(t)+c \dot{x}(t-\sigma)=b x(t-\sigma)
$$

is asymptotically stable if and only if

$$
\begin{aligned}
& 0<|c|<1, \\
& b<0, \\
& \sigma<\sqrt{\frac{1-c^{2}}{b^{2}}} \arccos (-c) .
\end{aligned}
$$

Based on Lemma 7, we have the following explicit result.

Corollary 8. Let $G=(\mathscr{V}, \mathscr{E})$ be an undirected connected weighted graph. The neutral multiagent system

$$
\dot{X}(t)+c \dot{X}(t-\sigma)=\Delta X(t-\sigma)
$$

can achieve average consensus if and only if $0<|c|<1$ and $\sigma<\bar{\tau}$, where

$$
\bar{\tau}=\sqrt{\frac{1-c^{2}}{\lambda_{1}^{2}}} \arccos (-c) .
$$


Proof. By Theorem 6, system (19) can achieve average consensus if and only if all the following scalar differential equations

$$
\dot{\alpha}_{k}(t)+c \dot{\alpha}_{k}(t-\sigma)=\lambda_{k} \alpha(t-\sigma), \quad k=1, \ldots, n-1
$$

are asymptotically stable. By Lemma 7, all equations (21) are asymptotically stable if and only if

$$
\begin{aligned}
& 0<|c|<1, \\
& \lambda_{k}<0, \\
& \sigma<\sqrt{\frac{1-c^{2}}{\lambda_{k}^{2}}} \arccos (-c), \\
& k=1, \ldots, n-1 .
\end{aligned}
$$

Noting that $\lambda_{k}<0$, for $k=1, \ldots, N-1$, all equations (21) are asymptotically stable if and only if

$$
\begin{aligned}
0 & <|c|<1, \\
\sigma & <\sqrt{\frac{1-c^{2}}{\lambda_{1}^{2}}} \arccos (-c) .
\end{aligned}
$$

The proof of Corollary 8 is completed.

3.2. Networks with Switching Topologies. Note that system (5) can be rewritten in the following matrix form:

$$
\dot{X}(t)+c \dot{X}(t-\sigma)=L(G) X(t-\tau(t)),
$$

where $L(G)$ and $\sigma$ are defined as above and the time-varying delay $\tau(t)$ satisfies the assumptions $(H) 0 \leq \tau(t) \leq h$ and $\dot{\tau}(t) \leq d$ with $h>0$ and $0 \leq d<1$.

We are here interested in discussing such a problem of whether a network with switching topology can still solve average consensus. In this case, the following switched system is considered:

$$
\begin{aligned}
\dot{X}(t)+c \dot{X}(t-\sigma)=L\left(G_{s}\right) X(t-\tau(t)) & \\
& s=\theta(t) \in \vartheta_{0},
\end{aligned}
$$

where $L\left(G_{s}\right)$ is the Laplacian matrix of the undirected graph $G_{s}=\left(\mathscr{V}_{s}, \mathscr{E}_{s}\right)$ that belongs to set $\Gamma$ and $\Gamma$ is a finite collection of graphs of order $n$ with an index set $\vartheta_{0} \subset Z$. The map $\theta(t)$ : $\mathbb{R} \rightarrow \vartheta_{0}$ is a switching signal that determines the network topology.

Definition 9. The neutral multiagent system (25) is said to achieve average consensus under arbitrary switching if each solution of system (25) satisfies $\lim _{t \rightarrow \infty} X(t)=\alpha \mathbf{1}$ with $\alpha=$ $(1 / n) \sum_{i=1}^{n} x_{i}(0)$

Let us first take the following reduced-order transformation:

$$
\begin{gathered}
y_{12}=x_{1}-x_{2}, \\
y_{13}=x_{1}-x_{3}, \\
\vdots \\
y_{1 n}=x_{1}-x_{n} .
\end{gathered}
$$

The system (25) can be transformed into the following reduced-order disagreement system:

$$
\dot{Y}(t)+c \dot{Y}(t-\sigma)=A_{s} Y(t-\tau(t)), \quad s=\theta(t) \in \vartheta_{0},
$$

where $Y=\left[y_{12}, y_{13}, \ldots, y_{1 n}\right]^{T}$ and $A_{s}=E L\left(G_{s}\right) F$ with

$$
\begin{aligned}
& E=\left(\begin{array}{cc}
\mathbf{1} & -I_{n-1}
\end{array}\right), \\
& F=\left(\begin{array}{c}
\mathbf{0}^{T} \\
-I_{n-1}
\end{array}\right) .
\end{aligned}
$$

Define the operator $\mathscr{B}: \mathbb{R} \rightarrow \mathbb{R}$ as

$$
\mathscr{B}\left(Y_{t}\right)=Y(t)+c Y(t-\sigma)
$$

The operator $\mathscr{B}$ is stable when $0<|c|<1$ and

$$
\dot{\mathscr{B}}\left(Y_{t}\right)=A_{s} Y(t-\tau(t))
$$
lemma.

Before giving the main result, we need the following

Lemma 10 (see [7]). For any real differentiable vector function $x(t) \in \mathbb{R}^{n}$ and any $n \times n$ constant matrix $W=W^{T}>0$, the following inequality holds:

$$
\begin{aligned}
& h^{-1} {[x(t)-x(t-\tau(t))]^{T} W[x(t)-x(t-\tau(t))] } \\
& \leq \int_{t-\tau(t)}^{t} \dot{x}^{T}(s) W \dot{x}(s) d s, \quad t \geq 0,
\end{aligned}
$$

where $\tau(t)$ satisfies $0 \leq \tau(t) \leq h$.

Theorem 11. Assume that $(H)$ holds and $0<|c|<1$. System (25) can achieve average consensus under arbitrary switching, if there exist symmetric positive definite matrices $P, Q, M, S$, and $R$ of appropriate dimensions such that the following linear matrix inequality holds:

$\Omega_{s}$

$$
\begin{aligned}
& =\left(\begin{array}{cccc}
-Q+S+M & P A_{s}+Q & 0 & 0 \\
A_{s}^{T} P+Q & \Upsilon & c A_{s}^{T} P & -c h^{2} A_{s}^{T} Q-c A_{s}^{T} R \\
0 & c P A_{s} & -S & 0 \\
0 & -c h^{2} Q A_{s}-c R A_{s} & 0 & -R+c^{2} h^{2} Q+c^{2} R
\end{array}\right) \\
& <0, \quad s \in 9_{0},
\end{aligned}
$$


Proof. In spite of the existence of switching topology, similar to the discussion given in Remark 3, system (25) achieves average consensus once it solves consensus. Therefore, based on the above analysis, it is sufficient to prove that system (27) is asymptotically stable. Let $V(t)=V_{1}(t)+V_{2}(t)+V_{3}(t)+$ $V_{4}(t)+V_{5}(t)$ be the common Lyapunov function for system (27), where

$$
\begin{aligned}
& V_{1}(t)=\mathscr{B}\left(Y_{t}\right)^{T} P \mathscr{B}\left(Y_{t}\right), \\
& V_{2}(t)=h \int_{t-h}^{t}(h-t+\xi) \dot{Y}^{T}(\xi) Q \dot{Y}(\xi) d \xi \\
& V_{3}(t)=\int_{t-\tau(t)}^{t} Y^{T}(\xi) M Y(\xi) d \xi \\
& V_{4}(t)=\int_{t-\sigma}^{t} Y^{T}(\xi) S Y(\xi) d \xi \\
& V_{5}(t)=\int_{t-\sigma}^{t} \dot{Y}^{T}(\xi) R \dot{Y}(\xi) d \xi .
\end{aligned}
$$

Assume that the sth subsystem is activated at time $t$; that is, $\theta(t)=s$. In the sequel, by considering the right-hand side derivative of $V(t)$, that is, $D_{+} V(t)$, along the trajectory of the solution of system (27), we have

$$
\begin{aligned}
D_{+} V_{1}(t)= & 2 \mathscr{B}\left(Y_{t}\right)^{T} P \dot{\mathscr{B}}\left(Y_{t}\right) \\
= & 2 Y^{T}(t) P A_{s} Y(t-\tau(t)) \\
& +2 c Y^{T}(t-\tau(t)) A_{s}^{T} P Y(t-\sigma), \\
D_{+} V_{2}(t) \leq & h^{2} \dot{Y}^{T}(t) Q \dot{Y}(t) \\
& -h \int_{t-\tau(t)}^{t} \dot{Y}^{T}(\xi) Q \dot{Y}(\xi) d \xi .
\end{aligned}
$$

Noting that $\dot{Y}(t)=A_{s} Y(t-\tau(t))-c \dot{Y}(t-\sigma)$ and following Lemma 10, we have

$$
\begin{aligned}
D_{+} V_{2}(t) \leq & h^{2} Y^{T}(t-\tau(t)) A_{s}^{T} Q_{s} Y(t-\tau(t)) \\
& -2 c h^{2} Y^{T}(t-\tau(t)) A_{s}^{T} Q \dot{Y}^{T}(t-\sigma) \\
& +c^{2} h^{2} \dot{Y}^{T}(t-\sigma) Q \dot{Y}(t-\sigma) \\
& -Y^{T}(t) Q Y(t)+2 Y^{T}(t) Q Y(t-\tau(t)) \\
& -Y^{T}(t-\tau(t)) Q Y(t-\tau(t)) .
\end{aligned}
$$

In addition,

$$
\begin{aligned}
D_{+} V_{3}(t) \leq & Y^{T}(t) M Y(t) \\
& -(1-d) Y^{T}(t-\tau(t)) M(t-\tau(t)), \\
D_{+} V_{4}(t)= & Y^{T}(t) S Y(t)-Y^{T}(t-\sigma) S Y(t-\sigma), \\
D_{+} V_{5}(t)= & \dot{Y}^{T}(t) R \dot{Y}(t)-\dot{Y}^{T}(t-\sigma) R \dot{Y}(t-\sigma) \\
= & Y^{T}(t-\tau(t)) A_{s}^{T} R A_{s} Y(t-\tau(t)) \\
& -2 c Y^{T}(t-\tau(t)) A_{s}^{T} R \dot{Y}^{T}(t-\sigma) \\
& +c^{2} \dot{Y}^{T}(t-\sigma) R \dot{Y}(t-\sigma) \\
& -\dot{Y}^{T}(t-\sigma) R \dot{Y}(t-\sigma) .
\end{aligned}
$$

Therefore,

$$
\begin{aligned}
& D_{+} V(t) \leq Y^{T}(t)[-Q+S+M] Y(t)+2 Y^{T}(t) \\
& \cdot\left(P A_{s}+Q\right) Y(t-\tau(t))+Y^{T}(t-\tau(t)) \\
& \cdot\left[-Q-(1-d) M+h^{2} A_{s}^{T} Q A_{s}+A_{s}^{T} R A_{s}\right] \\
& \cdot Y(t-\tau(t))-Y^{T}(t-\sigma) S Y(t-\sigma) \\
& +2 c Y^{T}(t-\tau(t)) A_{s}^{T} P Y(t-\sigma) \dot{Y}^{T}(t-\sigma) \\
& \cdot\left(-R+c^{2} h^{2}+c^{2} R\right) \dot{Y}(t-\sigma)-2 c Y^{T}(t-\tau(t)) \\
& \cdot\left(h^{2} A_{s}^{T} Q+A_{s}^{T} R\right) \dot{Y}(t-\sigma) .
\end{aligned}
$$

Then, a new bound of $\dot{V}$ is given as follows:

$$
D_{+} V(t) \leq U \Omega U,
$$

where $U=\left[Y^{T}(t), Y^{T}(t-\tau(t)), Y^{T}(t-\sigma), \dot{Y}^{T}(t-\sigma)\right]^{T}$, and $\Omega$ is defined by (32). Noting that the matrix $\Omega_{s}$ is negative definite, there exists $\eta_{s}>0$ such that $D_{+} V(t) \leq-\eta_{s}\|Y(t)\|^{2}$. Let $\eta=$ $\min \left\{\eta_{s}: s \in \vartheta_{0}\right\}$. Then, for any switching signal, the zero solution of (27) is asymptotically stable since the operator $\mathscr{B}$ is stable. Thus, for any time-varying delay $\tau(t)$ satisfying $(H)$, system (25) achieves average consensus under any switching. The proof of Theorem 11 is completed.

When $d \geq 1$ or nothing has been known about the derivative of $\tau(t)$, construct the common Lyapunov function $V(t)=V_{1}(t)+V_{2}(t)+V_{4}(t)+V_{5}(t)$, where $V_{1}(t), V_{2}(t)$, $V_{4}(t)$, and $V_{5}(t)$ are defined as above. Similar to the proof of Theorem 11, we can easily obtain the following corollary.

Corollary 12. Assume that $0 \leq \tau(t) \leq h$ for some $h>0$ and $0<|c|<1$. System (25) achieves average consensus under arbitrary switching, if there exist symmetric positive definite 


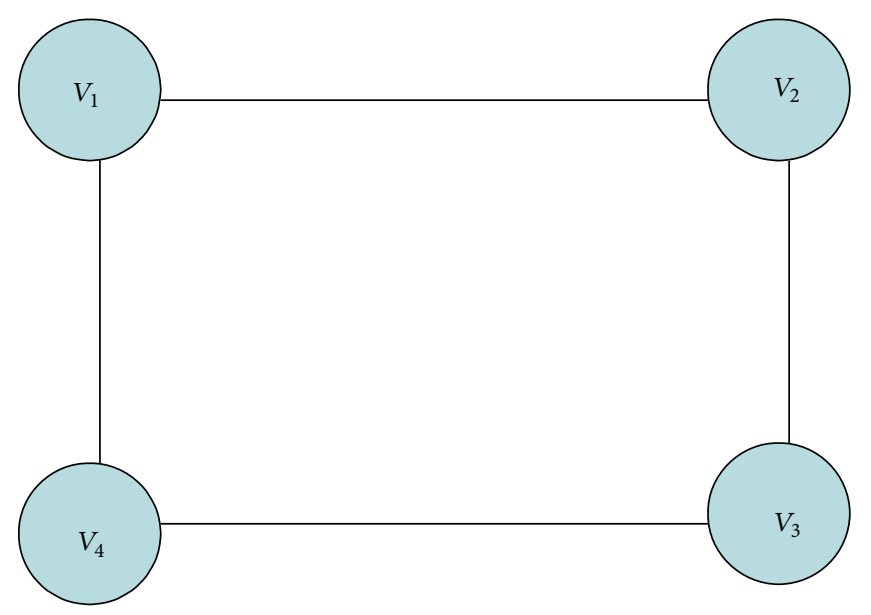

FIGURE 1: An undirected connected weighted graph.

TAble 1

\begin{tabular}{cccccccc}
\hline$d$ & 0 & 0.1 & 0.2 & 0.3 & 0.4 & 0.5 & Nothing \\
\hline$h$ & 0.3431 & 0.3337 & 0.3249 & 0.3162 & 0.3081 & 0.2975 & 0.2612 \\
\hline
\end{tabular}

$$
\Omega=\left(\begin{array}{cc}
-Q+S & P A_{s}+Q \\
A_{s}^{T} P+Q & -Q+h^{2} A_{s}^{T} Q A_{s}+A_{s}^{T} R A_{s} \\
0 & c P A_{s} \\
0 & -c h^{2} Q A_{s}-c R A_{s}
\end{array}\right.
$$

Remark 13. In Theorem 11 and Corollary 12, average consensus of the neutral multiagent system is determined by a linear matrix inequality rather than the connectivity of each switching topology. Similar to the analysis given in [7], it is not surprising that the connectivity of each switching topology is necessary to guarantee the feasibility of linear matrix inequalities (32) and (39).

Remark 14. Theorem 11 can also be extended to the case of directed graphs. Following the proof of Theorem 11, if each switching topology is a directed graph, the given linear matrix inequalities (32) guarantee consensus of the neutral multiagent system (25).

\section{Examples}

We now work out two examples to illustrate the theoretical results in this paper.

Example 1. Consider the neutral multiagent system (19) with the topology given by Figure 1.

The Laplacian matrix $L(G)$ takes the form

$$
L(G)=\left(\begin{array}{cccc}
-2 & 1 & 0 & 1 \\
1 & -2 & 1 & 0 \\
0 & 1 & -2 & 1 \\
1 & 0 & 1 & -2
\end{array}\right)
$$

matrices $P, Q, S$, and $R$ of appropriate dimensions such that the following linear matrix inequality holds:

We can get $\lambda_{1}=-4, \lambda_{2}=\lambda_{3}=-2$, and $\lambda_{4}=0$. When $c=0.45$, we have $\sigma<0.4549$ by Corollary 8 .

Example 2. Consider an undirected network with the following switching topologies shown in Figure 2.

Solving (32) gives the following estimate on $h$ for different $d$ and $c$, which guarantees system (25) associated with the switching topology given in Figure 2 achieves average consensus under arbitrary switching. When $c$ is fixed to 0.1 , the estimate on $h$ for different $d$ is shown in Table 1 .

When $d$ is fixed to 0.1 , the estimate on $h$ for different $c$ is shown in Table 2.

\section{Conclusion}

The average consensus problem of neutral multiagent systems in undirected networks with fixed and switching topologies is studied in this paper. Firstly, for the case of fixed topology, we establish sufficient and necessary conditions to average consensus by decoupling the neutral multiagent system in terms of the eigenvalues of the Laplacian matrix. Secondly, by using a linear matrix inequality method, average consensus criteria in terms of linear matrix inequality are given to determine the allowable upper bound of the time-varying communication delay for the case of switching topology. Finally, two examples are worked out to illustrate the theoretical results. 


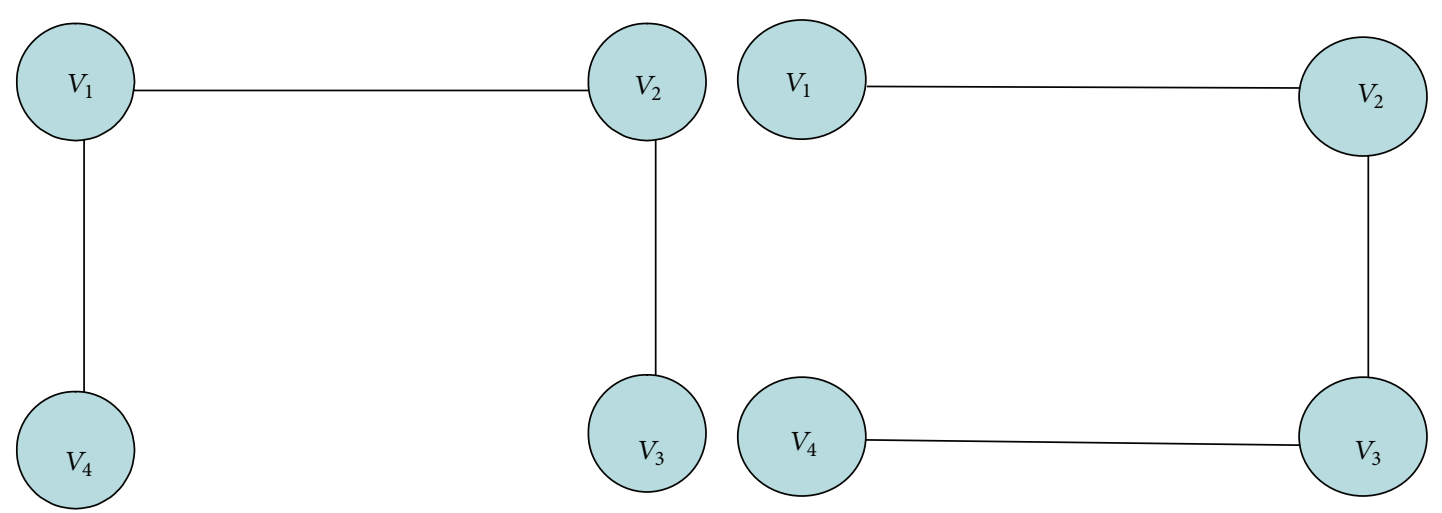

FIGURE 2: Two examples of undirected connected graphs.

TABLE 2

\begin{tabular}{ccccccc}
\hline$c$ & 0.1 & 0.2 & 0.3 & 0.4 & 0.5 & 0.6 \\
\hline$h$ & 0.3337 & 0.2798 & 0.2294 & 0.1816 & 0.1348 & 0.0874 \\
\hline
\end{tabular}

\section{Conflict of Interests}

The authors declare that there is no conflict of interests regarding the publication of this paper.

\section{Acknowledgments}

This work was supported by the Natural Science Foundation of Shandong Province under Grant no. JQ201119 and the National Natural Science Foundation of China under Grant nos. 61174217, 61374074, and 61473133.

\section{References}

[1] T. Vicsek, A. Czirók, E. Ben-Jacob, I. Cohen, and O. Shochet, "Novel type of phase transition in a system of self-driven particles," Physical Review Letters, vol. 75, no. 6, pp. 1226-1229, 1995.

[2] A. Jadbabaie, J. Lin, and A. S. Morse, "Coordination of groups of mobile autonomous agents using nearest neighbor rules," IEEE Transactions on Automatic Control, vol. 48, no. 6, pp. 988-1001, 2003.

[3] R. Olfati-Saber and R. M. Murray, "Consensus problems in networks of agents with switching topology and time-delays," IEEE Transactions on Automatic Control, vol. 49, no. 9, pp. 15201533, 2004.

[4] W. Ren and R. W. Beard, "Consensus seeking in multi-agent systems under dynamically changing interaction topologies," IEEE Transactions on Automatic Control, vol. 50, no. 5, pp. 655661, 2005.

[5] L. Wang and F. Xiao, "A new approach to consensus problems in discrete-time multiagent systems with time-delays," Science in China, Series F: Information Sciences, vol. 50, no. 4, pp. 625635,2007

[6] F. Xiao and L. Wang, "State consensus for multi-agent systems with switching topologies and time-varying delays," International Journal of Control, vol. 79, no. 10, pp. 1277-1284, 2006.
[7] Y. G. Sun, L. Wang, and G. M. Xie, "Average consensus in networks of dynamic agents with switching topologies and multiple time-varying delays," Systems \& Control Letters, vol. 57, no. 2, pp. 175-183, 2008.

[8] Y. G. Sun, "Average consensus in networks of dynamic agents with uncertain topologies and time-varying delays," Journal of the Franklin Institute, vol. 349, no. 3, pp. 1061-1073, 2012.

[9] M. Yu, L. Li, G. Xie, and H. Shi, "Average consensus in multiagent systems with the problem of packet losses when using the second-order neighbors' information," Mathematical Problems in Engineering, vol. 2014, Article ID 304126, 6 pages, 2014.

[10] X. Liu, T. Chen, and W. Lu, "Consensus problem in directed networks of multi-agents via nonlinear protocols," Physics Letters A, vol. 373, no. 35, pp. 3122-3127, 2009.

[11] J. Hu, J. Cao, J. Yu, and T. Hayat, "Consensus of nonlinear multiagent systems with observerbased protocols," Systems \& Control Letters, vol. 72, pp. 71-79, 2014.

[12] W. Ren and E. Atkins, "Distributed multi-vehicle coordinated control via local information exchange," International Journal of Robust and Nonlinear Control, vol. 17, no. 10-11, pp. 1002-1033, 2007.

[13] J. Zhu, Y.-P. Tian, and J. Kuang, "On the general consensus protocol of multi-agent systems with double-integrator dynamics," Linear Algebra and Its Applications, vol. 431, no. 5-7, pp. 701-715, 2009.

[14] Y.-P. Tian and C.-L. Liu, "Robust consensus of multi-agent systems with diverse input delays and asymmetric interconnection perturbations," Automatica, vol. 45, no. 5, pp. 1347-1353, 2009.

[15] W. Yu, G. Chen, M. Cao, and J. Kurths, "Second-order consensus for multi-agent systems with directed topologies and nonlinear dynamics," IEEE Transactions on Systems, Man, and Cybernetics, Part B: Cybernetics, vol. 40, no. 3, pp. 881-891, 2010.

[16] Q. Song, J. Cao, and W. Yu, "Second-order leader-following consensus of nonlinear multi-agent systems via pinning control," Systems \& Control Letters, vol. 59, no. 9, pp. 553-562, 2010.

[17] A. Abdessameud and A. Tayebi, "On consensus algorithms design for double integrator dynamics," Automatica, vol. 49, no. 1, pp. 253-260, 2013.

[18] Y. Zheng, Y. Zhu, and L. Wang, "Consensus of heterogeneous multi-agent systems," IET Control Theory and Applications, vol. 5, no. 16, pp. 1881-1888, 2011. 
[19] A. Bensoussan and J.-L. Menaldi, "Difference equations on weighted graphs," Journal of Convex Analysis, vol. 12, no. 1, pp. 13-44, 2005.

[20] H. S. Ren and Z. X. Zheng, "Algebraic Criterion for asymptotic stability of neutral equations of the form $\dot{x}(t)+c \dot{x}(t-\tau)+a x(t)+$ $b x(t-\tau)=0$," Acta Mathematica Sinica, vol. 42, no. 6, pp. 10771088, 1999. 


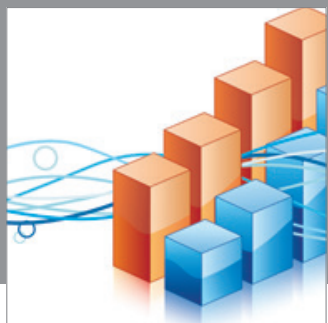

Advances in

Operations Research

mansans

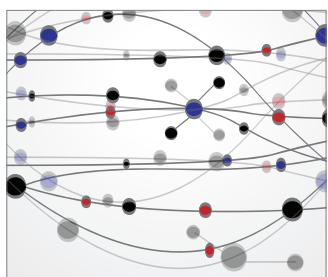

The Scientific World Journal
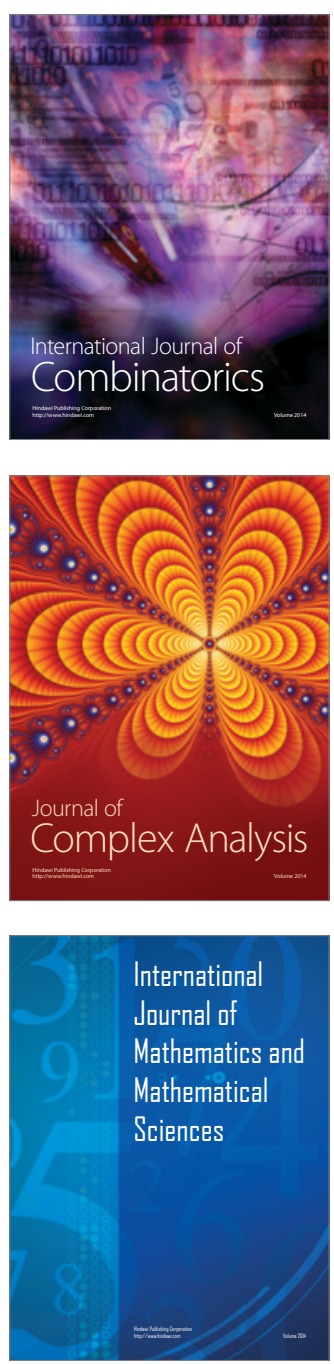
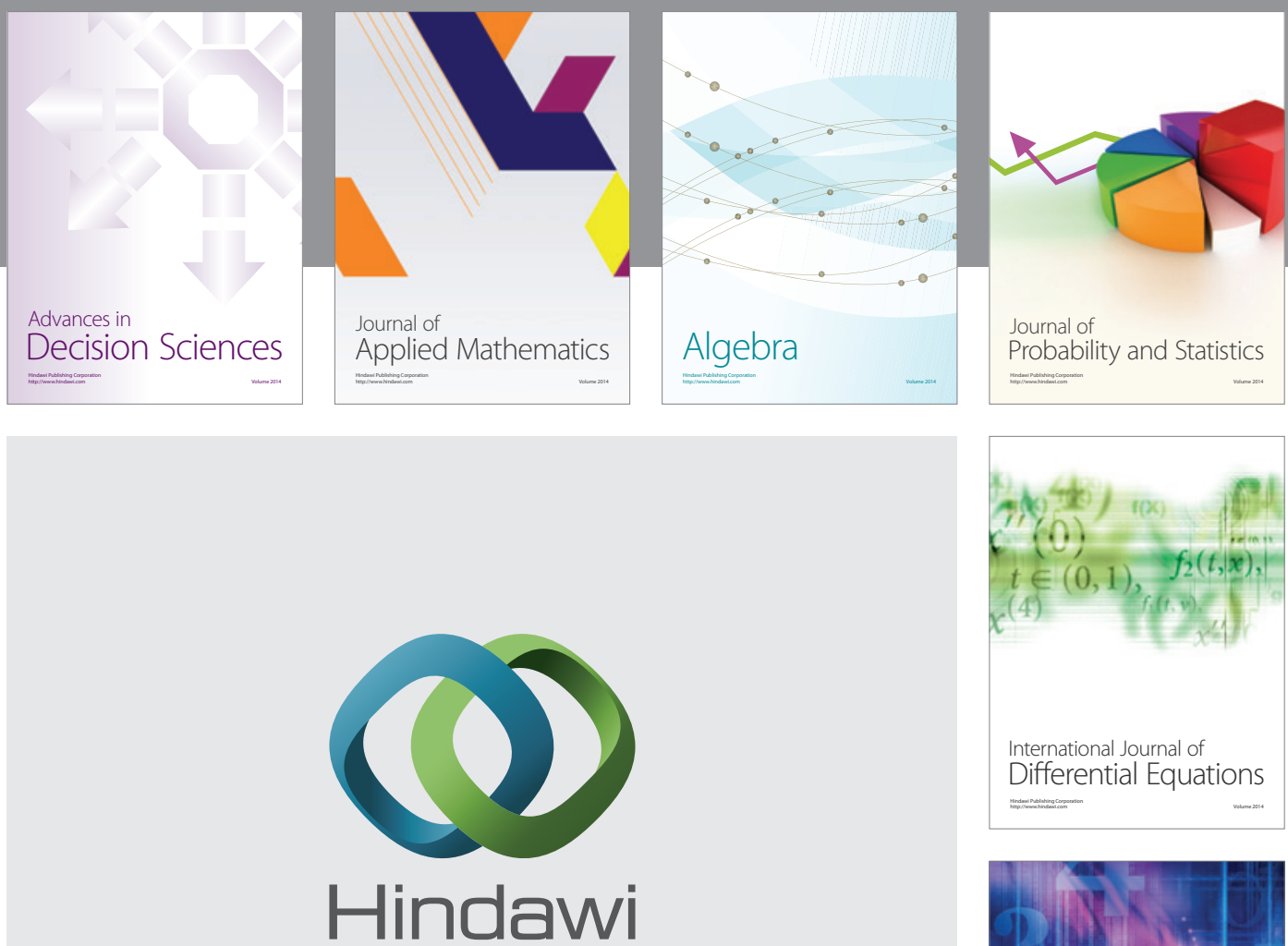

Submit your manuscripts at http://www.hindawi.com
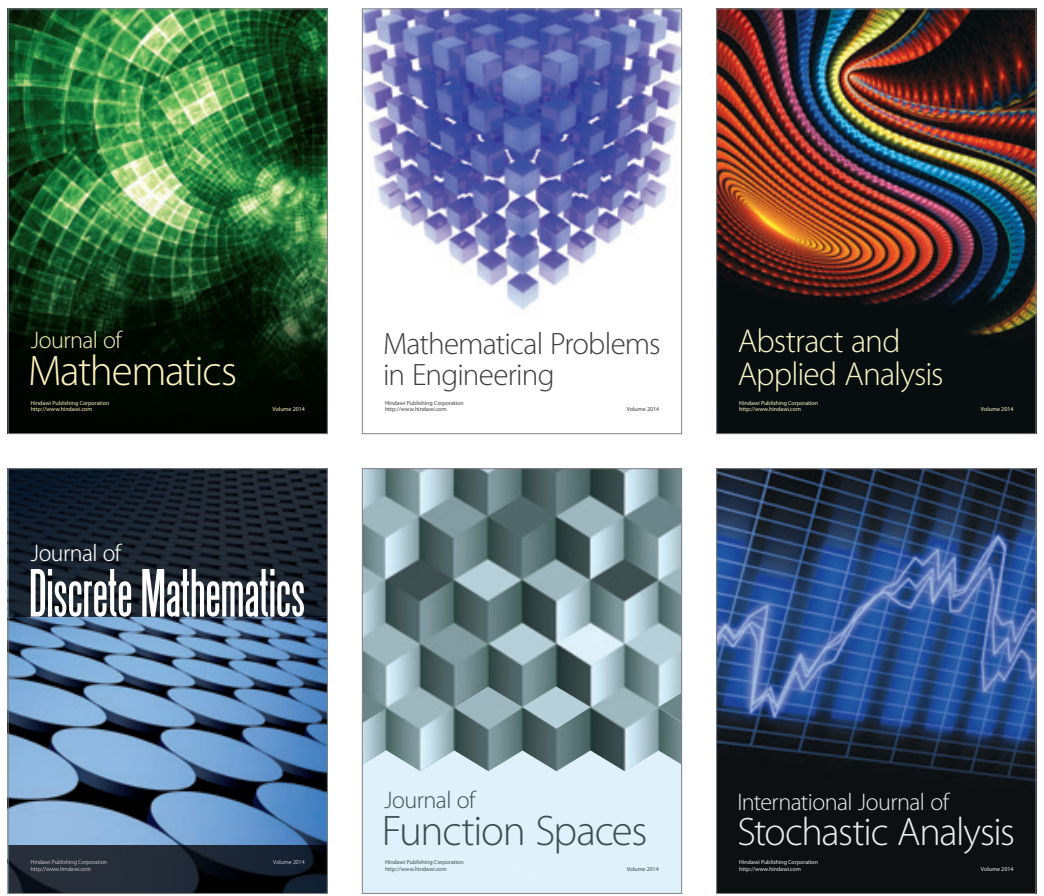

Journal of

Function Spaces

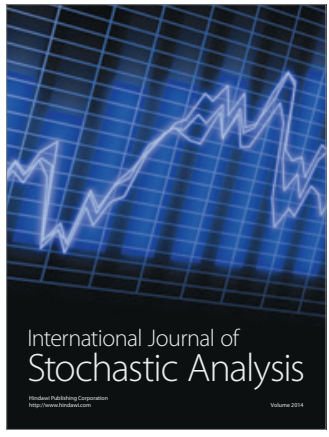

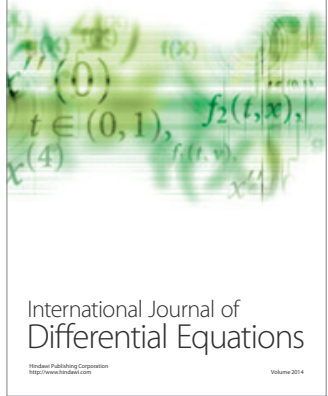
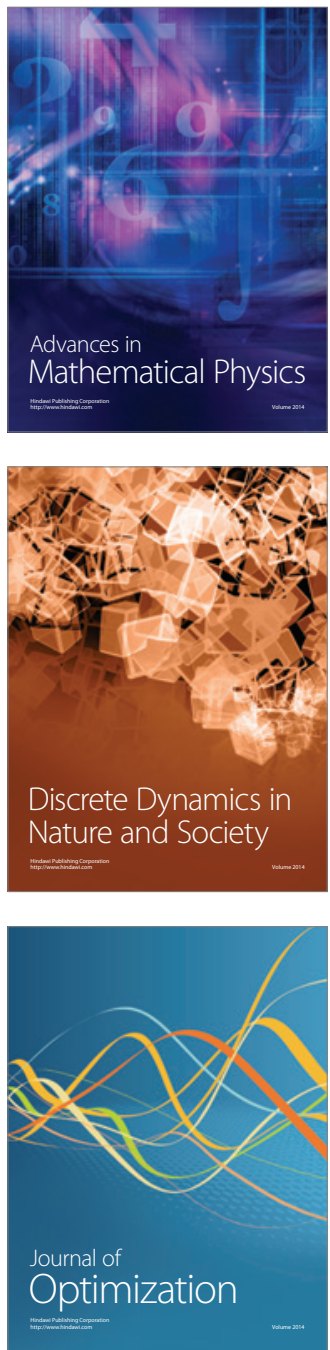